\title{
An AES - based Robust Image Encryption Scheme
}

\author{
Supriyo De \\ Dept. of ECE and AEIE, \\ Saroj Mohan Institute of Technology, Guptipara, \\ Hooghly, West Bengal, India, Pin-712512
}

\author{
Jaydeb Bhaumik \\ Dept. of ECE, \\ Haldia Institute of Technology, Haldia, \\ Purba Medinipur, West Bengal, India, Pin-721657
}

\begin{abstract}
The security of digital information has become a major issue during the last three decades. Encryption is one of the ways to ensure confidentiality for the digital data. Protection of multimedia data is now becoming a big challenge to create a healthy digital world. A large number of algorithms for data and image encryption are reported in the literature. Unfortunately, all traditional cryptosystems can't fulfill all the requirements of image encryption. Cryptographic weakness or high computational cost does not fulfill the real time requirement for the encryption technique. In this paper a novel approach is developed to encrypt the digital image which ensures better security with optimum cost. A linear transformation is done before encrypting an image by the Advanced Encryption Standard (AES) in ECB mode. The results show that the correlation between adjacent image elements is significantly reduced by employing the proposed scheme compared to other schemes. The histogram, correlation, entropy analysis as well as differential cryptanalysis of proposed image encryption scheme have been done to justify the strength of the proposed scheme.
\end{abstract}

\section{General Terms}

Security, Image Encryption

\section{Keywords}

Block Cipher, Advanced Encryption Standard, Linear transformation, Security analysis

\section{INTRODUCTION}

Image and video encryption have a huge application in various field including Internet communication, multimedia systems, medical imaging, telemedicine, confidential videoconferencing, military and defense maps and data-base, mobile computing, design of electronic circuits, technical model designing etc. Two parameters are mainly defining the effectiveness of image encryption. The first one is the speed of the encryption algorithm which important for online applications and the second one is the security of the encryption technique which is generally getting the importance in banking, defense sector. In this paper, encryption algorithm that can satisfy the requirements of these two parameters were implemented.

The Advance Encryption Standard (AES) was announced by the National Institute of Standard and Technology (NIST) in 2001 [1]-[3]. Basically, it includes three main processes, Key Expansion, Encryption and Decryption. In case of multimedia data security especially for digital image encryption by AES algorithm in ECB mode does not provide satisfactory result. For digital image there is a possibility where some positions pixel values do not have any variation. Since in ECB mode, each of same input blocks (length 128 bits) generate identical set of cipher blocks. The deficiency becomes more for those images where the color variation does not appear. In this deficiency pattern and shape of the images can be easily predicted from the cipher images.

Different techniques for image encryption have been studied in [4]. Combined encryption and compression scheme has also been reported by researchers in [5]. In [6] two direct approaches is implemented to prevent the deficiency. In first approach, the identical input difference is modified by adding different number sequences to them and the other approach is to remove the identical inputs using compression. Huang et al. [6] discussed limitation of AES in ECB mode for digital image encryption and proposed a modified AES for image encryption. In [7] authors proposed an algorithm based on AES key expansion in which the encryption process is a bit wise XOR operation of a set of image pixels along with the 128 bit key which changes for every set of pixels and it offers good resistance against brute force attack, key sensitivity tests and statistical cryptanalysis. Riad et al. [8] proposed a selective image encryption technique using hybrid chaos and block cipher. Shtewi et al. [9] also introduced a modified AES (MAES) for image encryption by changing ShiftRows operation of original AES. From Fig. 1(i), 1(j), 1(k) and 1(l), it is noted that scheme MAES in ECB mode also can't provide strong confidentiality for all images.

In this paper, we have proposed a new scheme called PE(Permutation followed by Encryption) to overcome the limitation of the AES and MAES. In this scheme we have introduced a permutation block before the AES block. The proposed scheme supports the parallel architecture of AES and also the complexity of permutation is very less compared to AES encryption scheme. In this work, the histogram, correlation and entropy analysis and resistance against differential attack of proposed image encryption scheme have been compared with the existing image encryption schemes. It is shown that encryption and decryption both can be done in parallel or pipelined mode. So, the proposed scheme is very much effective for multimedia application where high throughput is essential.

The rest of the paper is organized as follows. Section 2 discusses the proposed the PE algorithm for image encryption. Experimental results obtained by employing proposed scheme are elaborated in section 3 . In section 4 , security of proposed scheme has been evaluated. Also the scheme has been compared in terms of histogram analysis, correlation coefficient, entropy and differential cryptanalysis (Number of Pixels Change Rate and Unified Average Changing Intensity) with two existing schemes in this section and finally the paper is concluded in section 5 . 


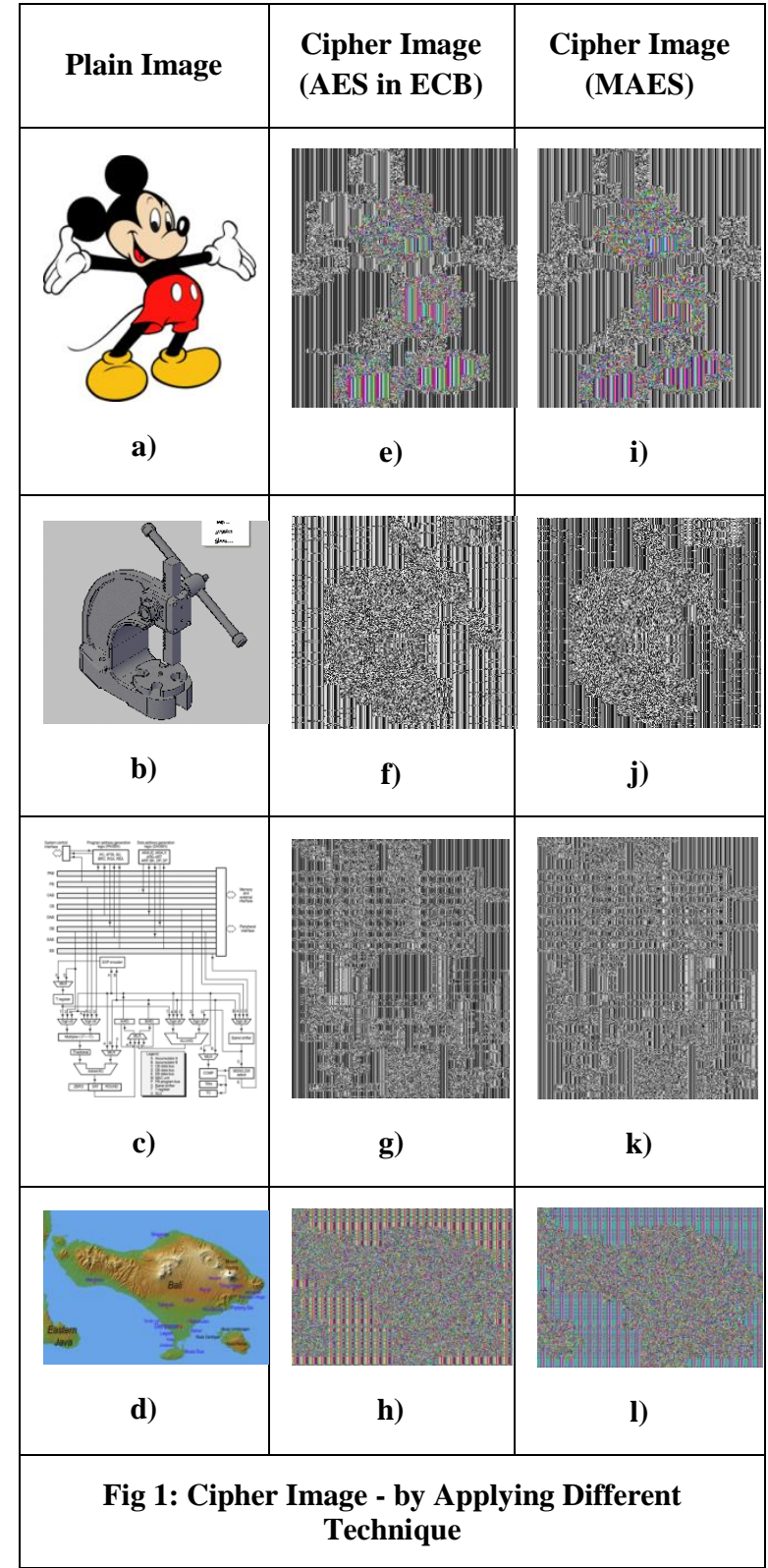

\section{PROPOSED SCHEME}

In this section, the proposed image encryption scheme based on permutation block (PB) followed by AES encryption has been introduced.

\subsection{Image Encryption}

Image encryption scheme introduced PB algorithm before the AES in ECB mode. PB performs a linear transformation of the plain image. In ECB mode of AES encryption scheme, a fixed key and fixed set of inputs always produce a same set of outputs and correspondingly the cipher image carries some information about the plain image. So, before applying the AES we introduced a permutation block with minimum complexity to shuffle the pixel values of the image to improve the security of encrypted image.

\subsubsection{Permutation Algorithm}

Permutation block is mainly incorporated with row shifting and column shifting of the image matrix with a linear relation. The total shifting process is done $\mathrm{N}$ (typically 10) times to get the better result. The operation of permutation block is briefly described below where ' $\mathrm{X}$ ' denotes the input matrix and ' $\mathrm{Y}$ ' is the shuffled output matrix. Row shift and column shift is totally a linear operation and this also depends upon the row number and column number respectively.

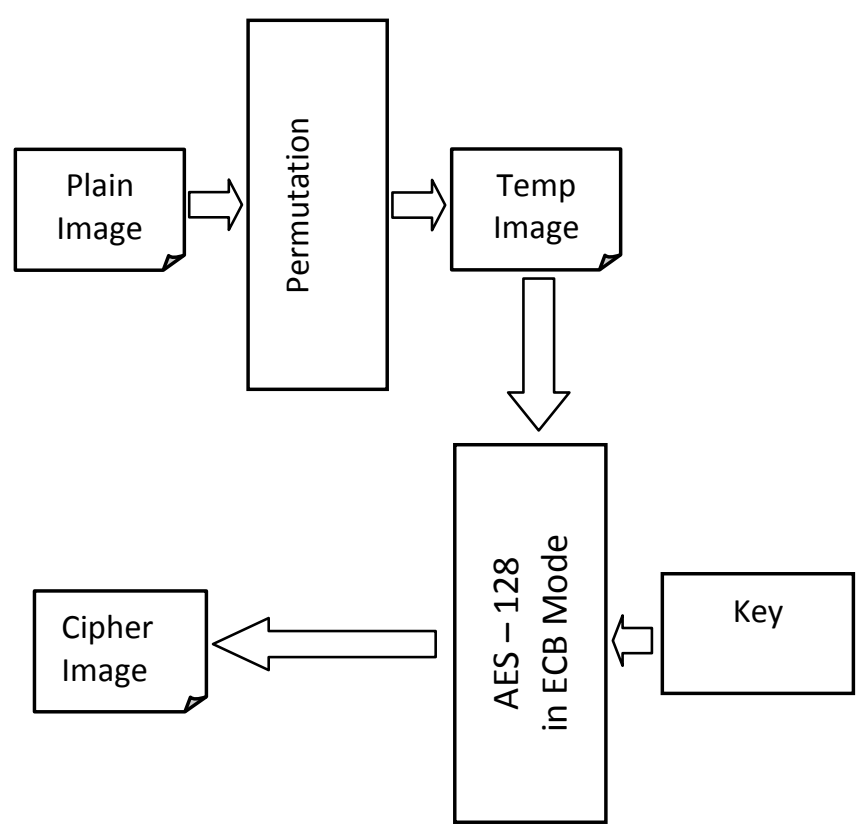

Fig 2: Block diagram of proposed image encryption scheme

$$
\begin{aligned}
& \text { Permutation Algorithm for each plane of colour Image } \\
& \text { Input: } \mathrm{X}_{(\mathrm{i})(\mathrm{j})} \\
& \text { Where, } \mathrm{i}=1,2,3,4 \ldots \ldots \mathrm{R} \text { (no. of row) } \\
& \qquad \mathrm{j}=1,2,3,4 \ldots \ldots \mathrm{C} \text { (no. of column) } \\
& \text { Output: } \mathrm{Y}_{(\mathrm{i})(\mathrm{j})} \\
& \text { Where, } \quad \mathrm{i}=1,2,3,4 \ldots \ldots \mathrm{R} \text { (no. of row) } \\
& \text { begin } \quad \mathrm{j}=1,2,3,4 \ldots \ldots \mathrm{C} \text { (no. of column) } \\
& \text { for } \mathrm{r}=1 \text { to } \mathrm{N} \\
& \text { for } \mathrm{i}=1 \text { to } \mathrm{R} \\
& \mathrm{X}_{(\mathrm{i})(\mathrm{j})}=\mathrm{Circular} \text { Right Shift Row (i) of } \mathrm{X}_{(\mathrm{i})(\mathrm{j})} \text { by } \mathrm{i} \text { bytes } \\
& \text { end } \\
& \text { for } \mathrm{j}=1 \text { to } \mathrm{C} \\
& \mathrm{X}_{(\mathrm{i})(\mathrm{j})}=\text { Circular Upward Shift Column }(\mathrm{j}) \text { of } \mathrm{X}_{(\mathrm{i})(\mathrm{j})} \text { by } \mathrm{j} \text { bytes } \\
& \text { end } \\
& \text { end } \\
& \mathrm{Y}_{(\mathrm{i})(\mathrm{j})}=\mathrm{X}_{(\mathrm{i})(\mathrm{j})} \\
& \text { end }
\end{aligned}
$$

\subsubsection{Encryption}

In the part of encryption AES is used in ECB mode. For AES encryption we have taken a fixed 128 bit key for a particular image. Figure 3 shows how the permutation block and AES 
block work together. Here 64 byte plain text is used for this example and 128 bit key for AES is shown in Fig. 3.

\begin{tabular}{|l|l|l|l|l|l|l|l|}
\hline FF & FF & FF & FF & FF & 80 & 80 & 80 \\
\hline FF & FF & FF & FF & 80 & 80 & 80 & 80 \\
\hline FF & FF & FF & FF & 32 & 32 & 37 & $3 \mathrm{C}$ \\
\hline FF & FF & FF & FF & FF & 80 & 50 & 2D \\
\hline 0A & 0A & 0A & 0A & 36 & $1 \mathrm{E}$ & $3 \mathrm{C}$ & $3 \mathrm{C}$ \\
\hline 0A & 0A & 0A & 0A & 0A & 4B & 56 & 23 \\
\hline 0A & 0A & 0A & 0A & 44 & 5F & 64 & B4 \\
\hline 0A & 0A & 0A & 0A & 0A & 31 & 19 & 0F \\
\hline \multicolumn{7}{|c|}{ Plain Image } \\
\hline
\end{tabular}

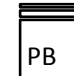

\begin{tabular}{|c|c|c|c|c|c|c|c|}
\hline 80 & $0 \mathrm{~A}$ & $\mathrm{FF}$ & FF & 56 & $\mathrm{FF}$ & $0 \mathrm{~A}$ & 36 \\
\hline $0 \mathrm{~A}$ & 80 & 80 & $0 \mathrm{~A}$ & FF & 31 & $3 \mathrm{C}$ & $\mathrm{FF}$ \\
\hline FF & FF & $0 \mathrm{~A}$ & 37 & $0 \mathrm{~A}$ & OA & 80 & 64 \\
\hline 80 & 23 & FF & $0 \mathrm{~A}$ & $1 \mathrm{E}$ & 80 & $0 \mathrm{~A}$ & $\mathrm{FF}$ \\
\hline $0 \mathrm{~A}$ & 32 & 19 & $0 \mathrm{~A}$ & $\mathrm{FF}$ & 44 & 50 & $\mathrm{FF}$ \\
\hline $3 \mathrm{C}$ & $0 \mathrm{~A}$ & $0 \mathrm{~A}$ & 80 & B4 & FF & FF & $4 B$ \\
\hline $0 \mathrm{~A}$ & $3 \mathrm{C}$ & $\mathrm{FF}$ & $0 \mathrm{~A}$ & $\mathrm{FF}$ & 80 & $0 \mathrm{~A}$ & $\mathrm{FF}$ \\
\hline $0 \mathrm{~A}$ & FF & $5 \mathrm{~F}$ & $2 \mathrm{D}$ & FF & $0 \mathrm{~A}$ & 32 & OF \\
\hline
\end{tabular}

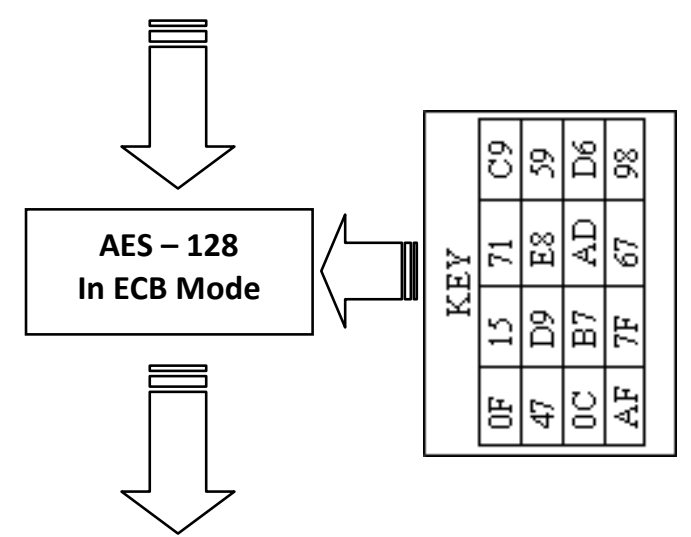

\begin{tabular}{|c|c|c|c|c|c|c|c|}
\hline 04 & DB & $\mathrm{C} 2$ & 82 & $\mathrm{CA}$ & 70 & A2 & $\mathrm{FE}$ \\
\hline 65 & 62 & 24 & B1 & $6 \mathrm{C}$ & OD & B0 & 51 \\
\hline 68 & DB & $9 \mathrm{~B}$ & 16 & 90 & $0 \mathrm{~A}$ & 1D & $4 \mathrm{C}$ \\
\hline 94 & B3 & F0 & $1 \mathrm{E}$ & $\mathrm{C} 8$ & 39 & $4 \mathrm{E}$ & $2 \mathrm{C}$ \\
\hline 20 & $\mathrm{AC}$ & 42 & 88 & 58 & 54 & 86 & 30 \\
\hline $1 \mathrm{E}$ & C6 & F0 & $3 \mathrm{C}$ & $0 \mathrm{E}$ & $\mathrm{C} 8$ & 67 & 95 \\
\hline $\mathrm{DE}$ & A9 & 41 & C6 & $3 \mathrm{D}$ & $8 \mathrm{D}$ & 74 & 58 \\
\hline $7 \mathrm{C}$ & C8 & 04 & $3 \mathrm{D}$ & 58 & $\mathrm{EC}$ & 27 & A1 \\
\hline \multicolumn{8}{|c|}{ Cipher Image } \\
\hline
\end{tabular}

Fig 3: Flow of Image Encryption Scheme

\subsection{Image Decryption}

Image decryption process follows the reverse sequence of the image encryption. The flow starts from the AES decryption in ECB mode and then the intermediate output is just goes under the reverse permutation block algorithm i.e. instead of Circular Right Shift Row and Circular Upward Shift Column here Circular Downward Shift Column and Circular Left Shift Row is used.

\section{EXPERIMENTAL RESULTS}

Experiments are performed using different plain images to prove the validity of the proposed algorithm. Figures 4(e), 4(f), 4(g) and 4(h) are the output of the PE algorithm and the corresponding original images are shown in Fig. 4(a), 4(b), 4(c) and 4(d). Output of permutation block doesn't provide any security because it is linear, reversible and no secret key is associated. This intermediate output is now applied as input of AES encryption scheme to obtain the cipher image. Figures 4(i), 4(j), 4(k) and 4(l) show the cipher image output of the proposed scheme.

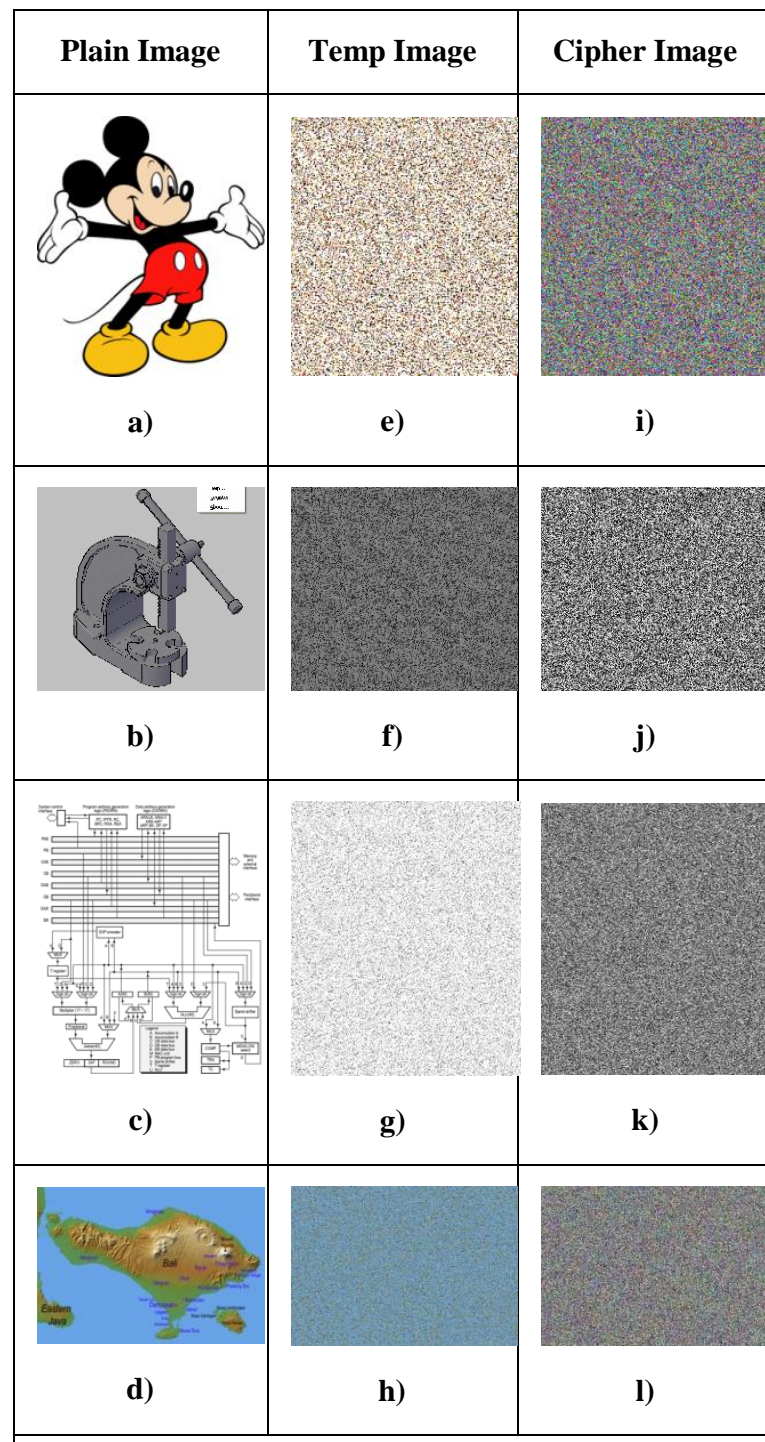

Fig 4: Image Encryption Result for Proposed Scheme

\section{SECURITY ANALYSIS}

Entropy, histogram and correlation are the three important parameters to analyze the security of image encryption algorithm. Entropy of encrypted image possibly increases through efficient encryption algorithm. On the other hand correlation between two adjacent pixels can be reduced and uniform histogram can be obtained in the encrypted image. A good encryption technique should be robust against cryptanalytic, statistical and brute-force attacks. 


\subsection{Key Space Analysis}

The key-space of an encryption technique is the set of possible keys that can be used to encode data using that technique. In the case of a strong encryption scheme, many keys must be tried in any brute-force attack on that technique. The proposed scheme has $2^{128}$ different combinations of the secret key. This long key space is sufficient for reliable practical use.

\subsection{Statistical Analysis}

Statistical analysis is essential to check the robustness/randomness of the cipher text obtained by the deterministic encryption scheme. Histogram and correlation of two adjacent pixels in the plain image/cipher image are analyzed to prove the effectiveness of the proposed scheme with respect to other well known encryption scheme.

\subsubsection{Histogram Analysis}

An image-histogram describes how the image-pixels are distributed by plotting the number of pixels at each intensity level. It is necessary for the cipher image to bear no statistical similarity. When the cipher image histogram is similar to the histogram of random image then it seems that the encryption algorithm has good performance. Figure 5 shows the histogram analysis of different plain images and cipher images. Figures 5(e), 5(f), 5(g) and 5(h) are histogram obtained by applying the AES encryption technique to the plain images and Figures 5(i), 5(j), 5(k) and 5(l) are histogram obtained by applying the MAES [9] encryption technique on the same plain images. The histogram of cipher images which are obtained by employing proposed scheme on same plain images are shown in Fig. 5(m), 5(n), 5(o) and 5(p). Histogram analysis of the proposed scheme justifies the effectiveness over MAES.

\subsubsection{Correlation Analysis}

The Correlation is one of the common and useful statistical tools to describe the degree of relationship between two sets of data. It is defined by the following equation.

$$
r=\frac{n \sum(x y)-\sum x y \sum y}{\sqrt{\left[n \sum x^{2}-\left(\sum x\right)^{2}\right]\left[n \sum y^{2}-\left(\sum y\right)^{2}\right]}} .
$$

Where,

$r$ : correlation value

$n$ : the number of pairs of data

इxy: sum of the products of paired data

$\Sigma x$ : sum of $x$ data

Iy: sum of y data

$\Sigma x^{2}:$ sum of squared $x$ data

$\Sigma y^{2}$ : sum of squared $y$ data

We have also analyzed the correlation between two adjacent pixels for plain image / cipher image respectively. Here we have taken the possible four neighboring angels for computing the correlation. Experimental result of correlation coefficient is shown in Table 1 to justify the effectiveness of our proposed technique. It breaks the correlation of the adjacent pixel in the cipher image. Figure 6 shows the pattern of correlation for plain images and corresponding cipher images obtained by AES, MAES and proposed encryption techniques. The result shows that the proposed scheme successfully breaks the correlation in between two neighbor pixels.

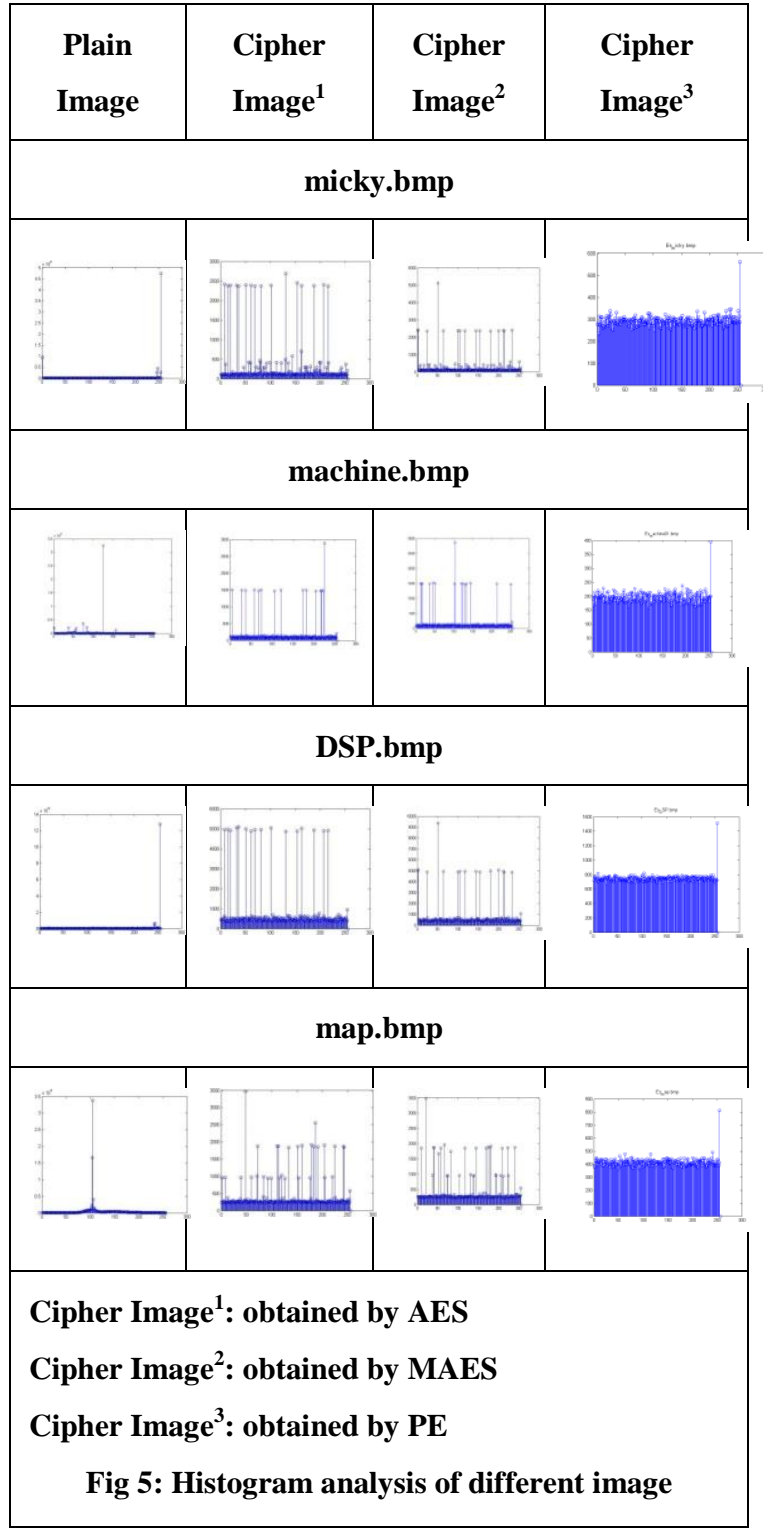

Table 1. Correlation coefficient of two adjacent pixels in the original and encrypted images

\begin{tabular}{|c|c|c|c|c|c|c|}
\hline 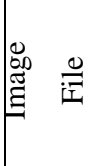 & ‡ี & 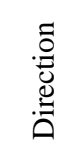 & $\begin{array}{l}\mathscr{\mathscr { O }} \\
\stackrel{\Xi}{\Xi} \\
\stackrel{\Xi}{\Xi} \\
\frac{\Xi}{2}\end{array}$ & 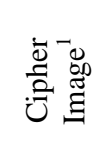 & 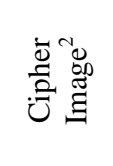 & 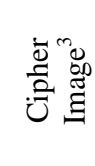 \\
\hline \multirow{4}{*}{ 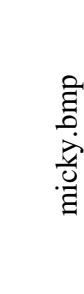 } & \multirow{4}{*}{ t: } & $0^{0}$ & 0.9510 & 0.0053 & -0.1045 & 0.0148 \\
\hline & & $45^{0}$ & 0.9253 & 0.0153 & -0.0856 & 0.0160 \\
\hline & & $90^{0}$ & 0.9523 & 0.5871 & 0.6171 & 0.0097 \\
\hline & & $135^{0}$ & 0.9151 & 0.0175 & -0.0850 & 0.0195 \\
\hline \multirow{3}{*}{$\frac{\stackrel{\Xi}{\Xi}}{\stackrel{\Xi}{\Xi}}$} & \multirow{3}{*}{ 芑 } & $0^{0}$ & 0.8403 & 0.0973 & -0.0348 & 0.0108 \\
\hline & & $45^{0}$ & 0.8165 & 0.0942 & -0.0145 & 0.0245 \\
\hline & & $90^{0}$ & 0.8638 & 0.4634 & 0.4072 & 0.0105 \\
\hline
\end{tabular}




\begin{tabular}{|c|c|c|c|c|c|}
\hline & $135^{0}$ & 0.8463 & 0.0938 & -0.0147 & 0.0274 \\
\hline \multirow{4}{*}{ 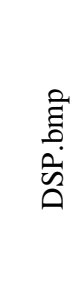 } & $0^{0}$ & 0.8086 & -0.0081 & -0.0422 & 0.0050 \\
\hline & $45^{0}$ & 0.4458 & -0.0034 & -0.0358 & 0.0167 \\
\hline & $90^{\circ}$ & 0.5820 & 0.4663 & 0.4808 & 0.0072 \\
\hline & $135^{0}$ & 0.4452 & -0.0052 & -0.0376 & 0.0156 \\
\hline \multirow{4}{*}{ 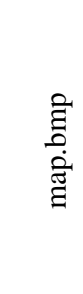 } & $0^{0}$ & 0.8533 & 0.0738 & 0.0079 & 0.0058 \\
\hline & $45^{0}$ & 0.8675 & 0.0569 & 0.0157 & 0.0167 \\
\hline & $90^{\circ}$ & 0.8830 & 0.2172 & 0.2365 & 0.0083 \\
\hline & $135^{0}$ & 0.7954 & 0.0566 & 0.0165 & 0.0167 \\
\hline \multicolumn{6}{|c|}{ Cipher Image $^{1}$ : obtained by AES } \\
\hline \multicolumn{6}{|c|}{ Cipher Image $^{2}$ obtained by MAES } \\
\hline \multicolumn{6}{|c|}{ Cipher Image $^{3}$ : obtained by PE } \\
\hline
\end{tabular}

\begin{tabular}{|c|c|c|c|}
\hline $\begin{array}{c}\text { Plain } \\
\text { Image }\end{array}$ & $\begin{array}{c}\text { Cipher } \\
\text { Image }^{1}\end{array}$ & $\begin{array}{c}\text { Cipher } \\
\text { Image }^{2}\end{array}$ & $\begin{array}{c}\text { Cipher } \\
\text { Image }^{3}\end{array}$ \\
\hline \multicolumn{2}{|c|}{ micky.bmp } \\
\hline \\
\hline
\end{tabular}

Cipher Image ${ }^{1}$ : obtained by AES

Cipher Image ${ }^{2}$ : obtained by MAES

Cipher Image ${ }^{3}$ : obtained by PE

Fig 6: Histogram analysis of different images 
otherwise, $D(i, j)=0$.

$W$ and H: no. of columns and rows of the image pixel values.

Experimental result of NPCR and UACI are shown in Table 3 and Table 4 respectively which established the consistency of the proposed scheme.

Table 3. NPCR for encrypted images

\begin{tabular}{|c|c|c|c|}
\hline \multirow{2}{*}{ Image File Name } & \multicolumn{3}{|c|}{ NPCR } \\
\cline { 2 - 4 } & AES & MAES & PE \\
\hline micky.bmp & 96.8258 & 99.8565 & 99.6281 \\
\hline machine.bmp & 99.6497 & 99.6398 & 99.6542 \\
\hline DSP.bmp & 97.3970 & 99.7479 & 99.6140 \\
\hline map.bmp & 99.7702 & 99.7185 & 99.6191 \\
\hline
\end{tabular}

Table 4. UACI for encrypted images

\begin{tabular}{|c|c|c|c|}
\hline \multirow{2}{*}{ Image File Name } & \multicolumn{3}{|c|}{ UACI } \\
\cline { 2 - 4 } & AES & MAES & PE \\
\hline micky.bmp & 11.6858 & 11.0891 & 16.7259 \\
\hline machine.bmp & 16.839 & 16.7375 & 16.6796 \\
\hline DSP.bmp & 13.1187 & 12.2807 & 16.7000 \\
\hline map.bmp & 14.8455 & 19.0599 & 16.6823 \\
\hline
\end{tabular}

\section{CONCLUSION}

In this paper a simple but reliable scheme has been proposed for image encryption using a combination of permutation followed by AES based encryption employing ECB mode. In terms of statistical analysis such as entropy, correlation and histogram the proposed scheme provides better results. In this scheme, encryption and decryption modules can work in parallel or/and pipelined mode which is very much effective for multimedia data encryption. In future it can be extended to video encryption as its required high throughput.

Till date AES is secure against all existing practical attacks. Since proposed PE is based on AES so it is secure. In spite of having the knowledge of inverse permutation on cipher image the scheme does not leak any information about plain image without decryption. Therefore, the proposed PE image encryption scheme is also secure against all existing practical attack.

\section{REFERENCES}

[1] J. Daemen and V. Rijmen, "The Design of Rijndael- AES, The Advanced Encryption Standard," Springer-Verlag, 2002.

[2] J. Daemen and V. Rijmen, "AES Proposal: Rijndael," Proc. of First Advanced Encryption Standard (AES) Conference, 1998.

[3] H. Yin, H. Debiao, K. Yong, and F. Xiande, "Highspeed ASIC implementation of AES supporting 128/192/256 bits," Proc. of Int. Conf. on Test and Measurement, IEEE, Vol. 1, Dec. 2009, pp. 95-98.

[4] R. Pakshwar, V. K. Trivedi, V. Richhariya, "A Survey on Different Image Encryption and Decryption Techniques," International Journal of Computer Science and Information Technologies, Vol. 4(1), 2013, pp. 113 116.

[5] X. Li, J. Knipe, H. Cheng, "Image Compression and Encryption Using Tree Structures," Elsevier Science Patten Recognition Letters, 1997, pp 1253-1259.

[6] C. W. Huang, Y. H. Tu, H. C. Yeh , S. H. Liu, C. J. Chang, "Image observation on the modified ECB operations in Advanced Encryption Standard," Proc. of Int. Conf. on Information Society (i-Society), London, Jun. 2011, pp. $264-269$.

[7] B. Subramanyan , V.M. Chhabria, T. G. S. Babu, "Image Encryption Based on AES Key Expansion," Emerging Applications of Information Technology (EAIT), Second International Conference, India,Feb. 2011, pp. $217-220$.

[8] A. M. Riad, A. H. Hussein, A. A. El-Azm, "A New Selective Image Encryption Approach Using Hybrid Chaos and Block Cipher," Proc. Of Int. Conf. on Informatics and Systems (INFOS), Cario, May. 2012, pp. 36-39.

[9] A. A. Shtewi, B. E. M. Hasan, A. E. F. A. Hegazy, "An Efficient Modified Advanced Encryption Standard (MAES) Adapted for Image Cryptosystems," International Journal of Computer Science and Network Security, Vol.10, Feb. 2010, pp. 226-232.

[10] Y. Wu, J. P. Noonan, S. Agaian, "NPCR and UACI Randomness Tests for Image Encryption," Cyber Journals: Multidisciplinary Journals in Science and Technology, Journal of Selected Areas in Telecommunications (JSAT), Apr. 2011.

[11] M. A. El-Wahed, S. Mesbah, A. Shoukry, "Efficiency and Security of Some Image Encryption Algorithms," Proc. of the World Congress on Engineering, Vol. 1, Jul. 2008. 